\title{
Forty-five years of development of the Luque segmental spinal instrumentation for the treatment of deformities and fractures of the thoracolumbar spine
}

\author{
Rodolfo Morales-Avalos*, José E. Baena-Trejo, Pedro M. Reyes-Fernández, \\ Oscar A. Martínez-Gutiérrez, Francisco I. Villarreal-Garcia, Luis A. Saavedra-Badillo, \\ José G. Mendoza-Mendoza, Ernesto C. Martinez-Avila, and Víctor M. Peña-Martínez \\ Spine Unit, Orthopedics and Traumatology Service, University Hospital "Dr. José Eleuterio González," Autonomous University of Nuevo Leon, \\ Monterrey, Nuevo Leon, Mexico
}

\begin{abstract}
In this review article, we present an overview of the Luque segmental spinal instrumentation, which was and continues to be one of the most commonly used methods for the treatment of paralytic scoliosis. The present article is center in the historical and social aspects in Mexico that boost the invention of the system, the previous anatomical and morphopathological studies that were carried out for the knowledge of the pathology and the understanding of it. Furthermore, the biomechanics and characteristics of the system, and its surgical technique, as well as the extension of its indications for use, a comparison between the Harrington method, which represents the standard of care in this pathology at the time, the diffusion of the technique around the world, the complications and limitations of its use, and the actual status of the technique. The Luque system is still taught in universities in Mexico to residents in training due to the persistence in some patients of a lack of social coverage, as well as extremely low social conditions, as some of the approximately 300 cases treated with this technique in our center since 1976.
\end{abstract}

Key words: Segmental spinal instrumentation. Luque instrumentation. Luque technique. Neuromuscular scoliosis. Luque system.

\section{Introduction}

\section{Development and concept}

The method of segmental fixation was first accomplished in 1972, by Dr. Javier Verdura, a neurosurgeon, for a fracture-dislocation of C3 and C4. Wires were passed underneath the laminae of both $\mathrm{C} 3$ and $\mathrm{C} 4$ to produce an instant "posterior fusion" with methyl methacrylate. Furthermore, due to the early experience of Harrington, methyl methacrylate was used to increase the area and solidity of the fixation ${ }^{1}$.
The concept of segmental spinal instrumentation was developed and published by Prof. Dr. Eduardo Luque Rebollar in Mexico City in $1973^{2}$. This arose as a response to the need to efficiently correct severe curves segmentally in patients with sequelae of polio and neuromuscular scoliosis, which had serious failures in maintaining the correction by the conventional methods of treatment in populations with extremely low socioeconomic resources which did not have access to orthodox treatment with corsets or orthoses, making surgical treatment in these patients imperative. This system allows

\section{Correspondence:}

*Rodolfo Morales-Avalos

E-mail: rodolfot59@ hotmail.com
Available online: 09-12-2019 DOI: 10.24875/RMU.19000093
Medicina Universitaria. 2019;21(4):145-151 www.medicinauniversitaria.org (http://creativecommons.org/licenses/by-nc-nd/4.0/). 
correction, immediate rigid fixation, and a high index of fusion while also providing the spinal cord the ability to develop adequate cardiopulmonary function by placing paravertebral metal longitudinal bars previously contoured and "L" shaped over the spine, which fix the vertebrae segmentally by knotting multiple double sublaminar wires (to decrease the risk of fracture), with one end joined by a bead and the other by a loop. Since the bars can be contoured in all planes, it is possible to correct deformities in the coronal plane by preserving the curvatures in the sagittal plane when correcting by transverse loads (especially in the apical area of the curve) applied individually in each vertebrae segment involved ${ }^{2}$ (Fig. 1).

In the same way, a certain derotation is also carried out in the transverse plane, because the wires on the concave side are tightened first. Fusion length must cover all deformities and include those vertebrae that are needed to establish compensatory curves and a well-balanced spine ${ }^{1}$.

In the original article, Dr. Luque concludes that segmental spinal instrumentation is a method of rigid internal fixation of the spine and is applicable to all surgical conditions of the spine where desirable with its use, any loss of correction is a technical error, no external fixation is needed, and correction is as good as or better than can be obtained with previous methods of treatment of scoliosis ${ }^{3}$.

\section{Previous anatomical studies}

This system was devised after carrying out morphopathological and biomechanical studies of the spine performed by Dr. Luque on cadavers, scoliotic bones, and in vivo studies ${ }^{4}$. Thus, concluding that the available correcting systems of the time were based on the functional response of the column, and not considering the bone deformity and anomalies in the soft tissues that also played a part in the correction of column deformities, and as a result, they represented an anatomical limitation in their techniques. He mentions that the spine, due to its segmental structure, should be fixated at every level if the goal is to obtain an internally rigid fixation'.

He considered the pathology as a complex deformity that combined several structural anomalies, such as lordosis, lateral deviation, and apical rotation, and that each of these anomalies required a specific anatomical correction. The lateral deviation is blocked by the shortening of all ligamentous and muscular structures on the concave side of the curve. Anatomical and clinical examples demonstrate overgrowth of the posterior structures on the convex side. Apical rotation is blocked by

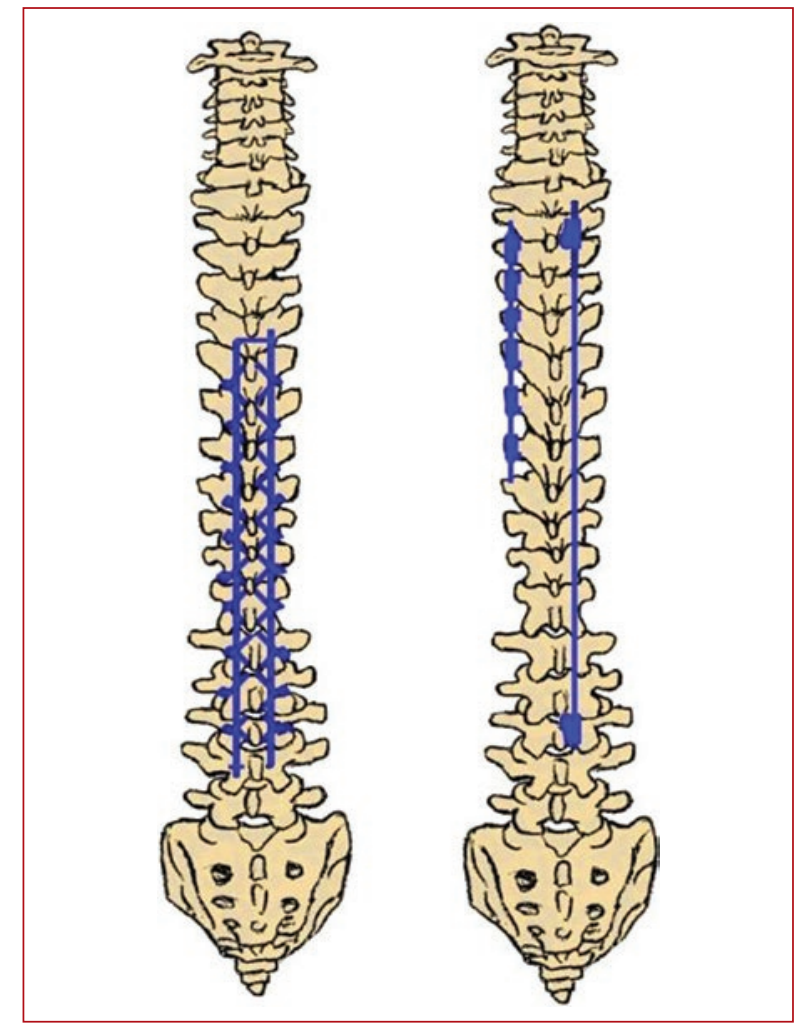

Figure 1. Representative outlines of Luque (left) and Harrington (right) instrumentation.

the locking of the facets on the concave side of the curve, as well as a contracture of the soft tissue on the same side, especially those attached to the transverse processes. The lordotic component is most probably limited by the vertebral body itself ${ }^{5}$. Most of these morphopathological concepts remain to this day.

\section{Biomechanics of the implant}

The spine, being a segmental structure, must be fixed at each level if rigid fixation is to be obtained. As the vertebral components move in flexion, extension, and lateral bends, they are complicated by rotation between each segment. A double paravertebral bar, making a triangle with the anterior supporting structures, discs, and vertebral body, gives better immobilization ${ }^{6}$. The study of the biomechanics of the implant that Dr. Luque designed and its adaptation to a spine with severe curvature established that the concave bar is weakest at the apex, where it receives transverse traction through the wires, and strongest at the ends of the curve, where it pushes against the end lamina to align with the spine. The convex bar is weakest at the ends of the curve, where the wires pull the flexible extremities into alignment, and strongest at the apex, where it acts as a point 
fulcrum over the most deformed laminae. By creating a couple force (uniting both bars), the weak point of one will be reinforced by the strong point of the other, and immobilization will be more rigid as a system?

Segmental instrumentation with sublaminar wires results in an even distribution of corrective forces with two lateral fixation points on each segment, which provides good rotary control. The rods apply pressure on the spine to correct the deformity. The L-rods and wire constructs aim at translation and coronal and sagittal balancing, rather than derotation, as their principle; hence, the extent of derotation is not the purpose that is intended to be achieved with this technique. Since there are multiple points of fixation with the Luque technique, the patient does not have to wear a brace after surgery. Therefore, segmental instrumentation with sublaminar wires has been widely adopted in the treatment of neuromuscular curves because it provides rigid fixation and allows for early mobilization without external support ${ }^{8}$.

The segmental spinal instrumentation designed by Dr. Luque produces an internal rigid fixation and alienation of the spine by fixing every vertebra in a threepoint system to the sagittal plane with bars and wires at a laminar level, using a disk as the third point of fixation. This technique is now extended for treatment in traumatic pathologies. Using "L" rods avoid migration and rotation of the hardware. "C" or "U" rods "Set" the length of the spine (when using an outrigger) and avoid a collapse in an unstable fracture ${ }^{9}$.

With the development of transversal tractions and the strong fixation that the Harrington instrumentation provided, two concepts were made clear that gave an advantage to the use of the Luque technique: (1) the more points to apply correcting forces, the less the force required at each fixation point and (2) if an arthrodesis is not made, it is possible to allow some growth of the spine, even when several operations are required to place longer bars ${ }^{6}$.

\section{Extension of the indications}

This cheap, low-profile, and effective segmental fixation method is, particularly apt for neuromuscular scoliosis since it provides load distribution in osteoporotic bones. Later, it was also applied to idiopathic curves, since its high primary stability omits the need for post-operative plaster or brace immobilization. Furthermore, the costs are lower than for pedicle screw constructs, making this approach superior to the classical Harrington method, which were impractical to perform on fragile osteoporotic bones such as those found in paralytic scoliosis or post-poliomyelitis, and prevented the growth of the spine due to the stepped bar and the hooks were anchored to each vertebra ${ }^{6}$.

This system was also used to produce a correction in a stable internal fixation in patients with spinal fractures (Figs. 2 and 3) and/or spinal instability due to metastatic disease (Fig. 4) or advanced degeneration of the spine, allowing early mobilization without the need for external post-operative immobilization ${ }^{6,10}$.

\section{Surgical technique}

The spine is exposed through a longitudinal incision in the middle of the back, to cover the entire deformity. After the appropriate hemostasis, subperiosteal dissection is carried out on all posterior elements of the spine, and the muscles are stripped subperiosteally up to the articular processes in the lumbar area and the costotransverse joints in the thoracic region on both sides ${ }^{11,12}$.

The following procedures are done in preparation for surgical site infection: (1) release of the contracted soft tissues on the concave side, including all ligaments; (2) destruction of the osseous deformity on the convex side at the apex, leaving an open convex wedge; (3) removal of all yellow ligaments in all spaces included in the deformity; (4) total facetectomies on both the concave and convex sides; and (5) creation of a posterolateral trough of bleeding bone over the position of the facetectomies, articular processes, and lateral lamina in the thoracic area. These posterolateral troughs are where bone chips are placed at the end of the procedure to produce a solid arthrodesis. In an attempt to avoid the narrowing of the neural canal reported with interlaminar arthrodesis and to obtain a better mechanical union, no decortication of the laminae is done. Segmental spinal instrumentation is carried out by slipping wire loops (No. $1.22 \mathrm{~mm}$ stainless 316, gauge wire) underneath the lamina at each vertebral level, cutting each loop, and leaving a wire on either side for the paravertebral bars. The rods (stainless steel 316) are three-sixteenths of an inch in diameter, $50 \mathrm{~cm}$ long, and cut to size ${ }^{6}$.

After partial removal of the ligamentum flavum to gain access to the spinal canal, the prevent wires are manually guided around the front side of the laminae through the space between the lamina and spinal cord. The two ends are passed around the rods and then tightened sequentially. As this mechanism is applied multisegmentally, the spine is gradually translated and bound to the rod $^{13}$.

The wires are threaded through the spinal canal at each vertebral level and are then twisted around the rods on each side of the spine. The wires are usually doubled (to 


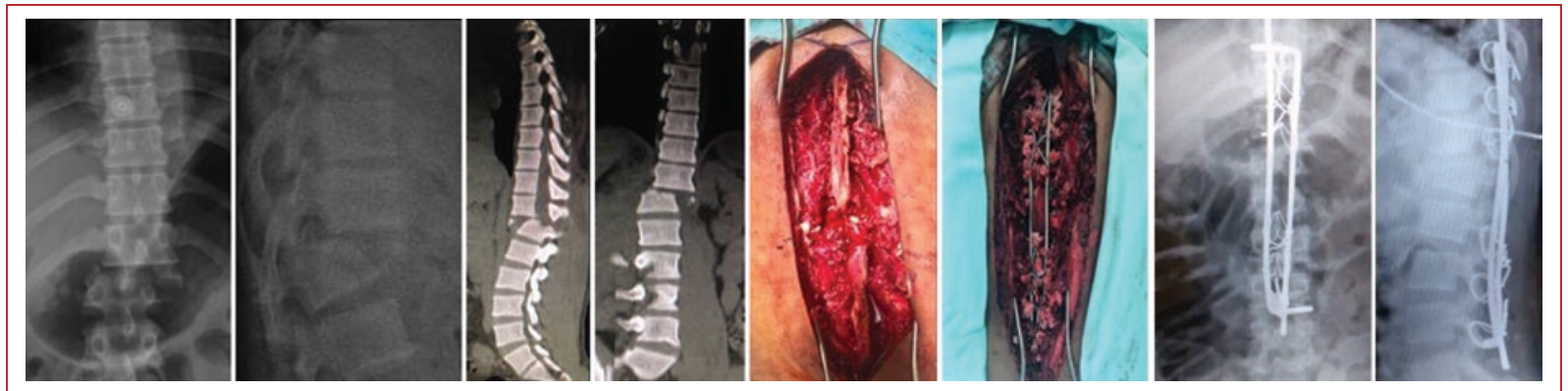

Figure 2. Case of a 32-year-old patient with a diagnosis of a luxation-fracture of $\mathrm{L} 1$ and subsequent wedging of $\mathrm{L} 1$ with an invasion of the medullary canal and complete neurological lesion. At the time of the surgery, a complete rupture of the supraspinatus ligament and lateral translation of the vertebra could be evidenced. An open reduction and instrumentation using the Luque system were performed three levels up and three levels down from the damage level.

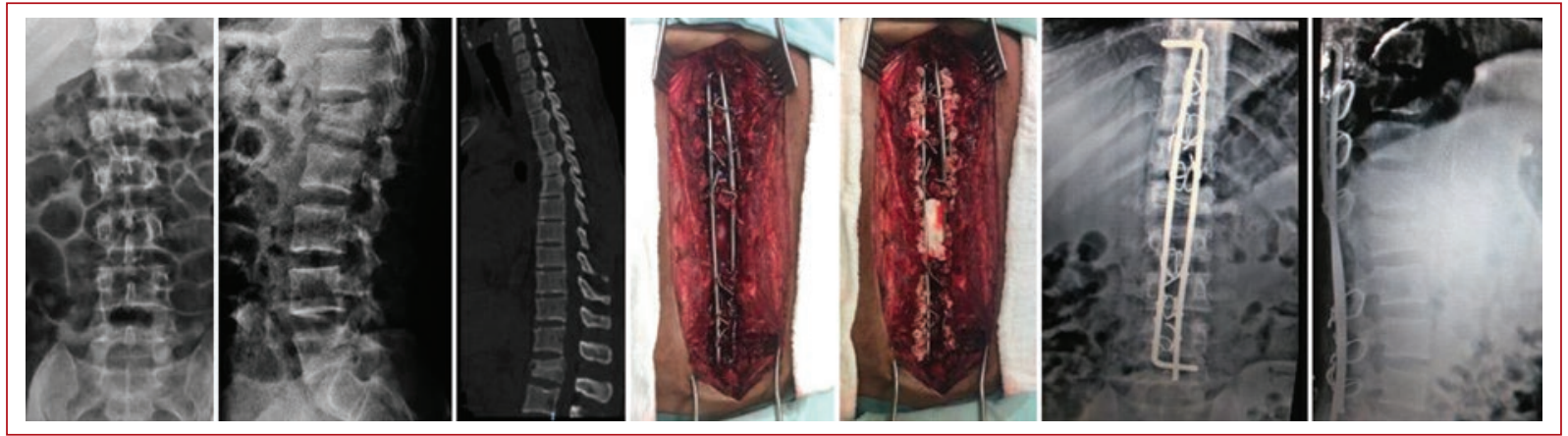

Figure 3. 32-year-old male patient with a diagnosis of a compression fracture of L1 with a partial neurological injury that was subjected to a segmental spinal Luque instrumentation. After the procedure, the patient showed an improvement in his neurological status.

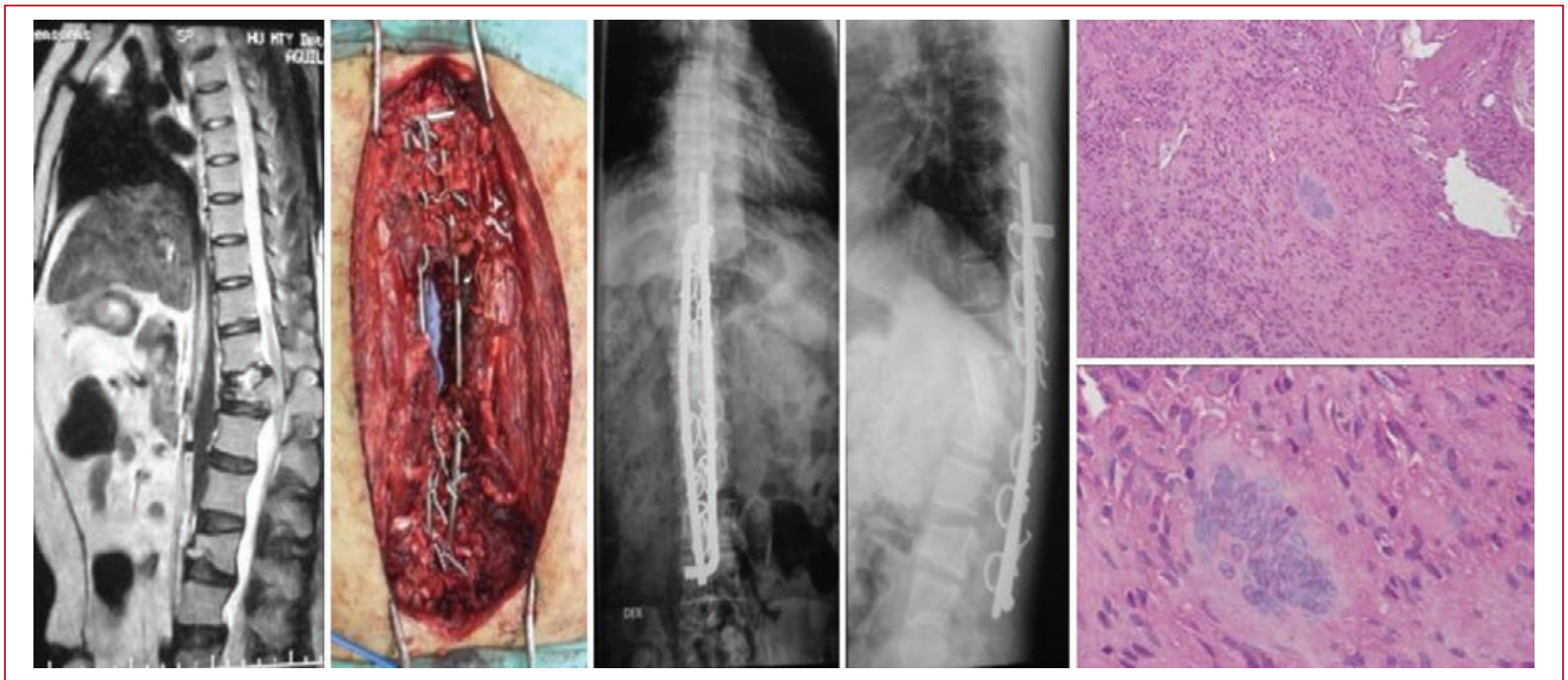

Figure 4. 37-year-old male patient with a diagnosis of spondylodiscitis at the T12 vertebra by Mycobacterium tuberculosis, which was treated with a T12 corpectomy, debridement and cleaning, fibular allograft placement, and the Luque segmental spinal instrumentation. The diagnosis was confirmed by histopathological means, where the formation of granulomas can be observed. 


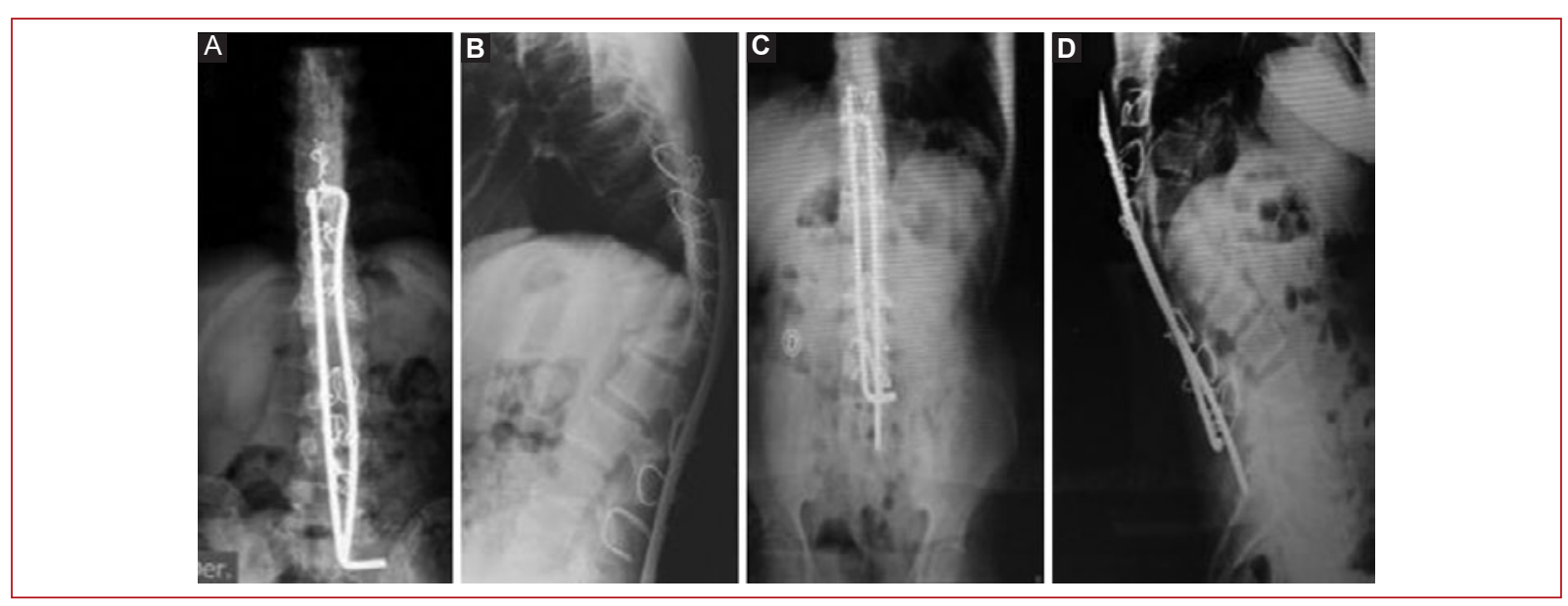

Figure 5. Example of complications observed in the follow-up of a post-operation patient of thoracolumbar vertebral fractures using Luque instrumentation. It is evidenced that a rupture of the wires leads to a failure in the system and loss of the alignment and reduction (A-D).

reduce the risk of fracturing the lamina), with one end joined by a bead and the other by a loop. The beaded end is contoured by creating a small bend at the tip that will emerge on the cephalad side of the lamina. A flatter contour minimizes intrusion into the canal. However, it is important to bear in mind that once metal wires are passed under the lamina, great care must be taken to ensure that none of the operating team inadvertently pushes one of the wires into the canal. To minimize this danger, each wire should be temporarily bent over the lamina ${ }^{14}$.

At the same time, and taking into account the maximum correction limits calculated in a preoperative manner, the bars are pre-molded to accept the residual deformity and the compensatory curve with the creation of an "L" angle at each end of the bar to prevent the migration of the bars. Parallel convex and concave rods are prevent to fit one cephalad and one caudal on either side of the spine ${ }^{1}$.

A small tunnel is made at the base of the spinous processes of the end vertebrae, or through the alae of the ilium immediately inferior to the posterosuperior spine, to introduce the "L" of the prevent bars. One bar is fixed on the cephalad portion of the deformity and the other on the caudal end. They are fixed independently to each vertebra by the previously placed wires under each lamina. In this way, a second-degree level arm is obtained with the convex rod, with pressure over the lamina at the apex and an aligning of the flexible end of the curve. Transverse traction is thus produced at the apex of the curve with the wires on the concave bar. On completion of the segmental correction, both bars are united with cross bands to make a couple force. The wire loop ends are cut and bent down away from the skin, and the wound is closed in the usual manner ${ }^{6}$.

\section{Diffusion of the technique}

The Luque method was adopted by national and international hospitals and has been used in multiple scientific works as a transcendental development in fixation systems. It has suffered multiple adaptations and modifications through the years, such as the placement of the wires now done in the spinous and transverse processes and the use of this technique in combination with others such as the Harrington or the anterior approach to perform a posterior fixation after the debridement or previous release ${ }^{15}$.

\section{Comparison with Harrington's method}

Harrington rods apply a longitudinal force on the spine; Luque rods apply transverse forces along sections of vertebrae. Harrington rods were the first accepted implant used to correct scoliosis in the early 1960s. The implant consists of a non-segmental system utilizing rods for distraction placed on the concave side of the curve, and another facultative rod placed on the convex side for compression ${ }^{16}$.

In Harrington rod instrumentation, metal rods and hooks are implanted along the outside of the spine. A long rigid distraction rod is placed on the concave side of the scoliotic curve. Along the convex side, a compression system of hooks and threaded semirigid rods may be added, especially if kyphosis is excessive ${ }^{16}$.

An average correction of $50 \%$, with a wide range reported from $28 \%$ to $63 \%$, can be achieved in adolescent idiopathic scoliosis. Limiting factors are that the efficiency of axial distraction is decreased as curve magnitude is decreased, and there is an increased risk of neurological complications with further distraction. External 


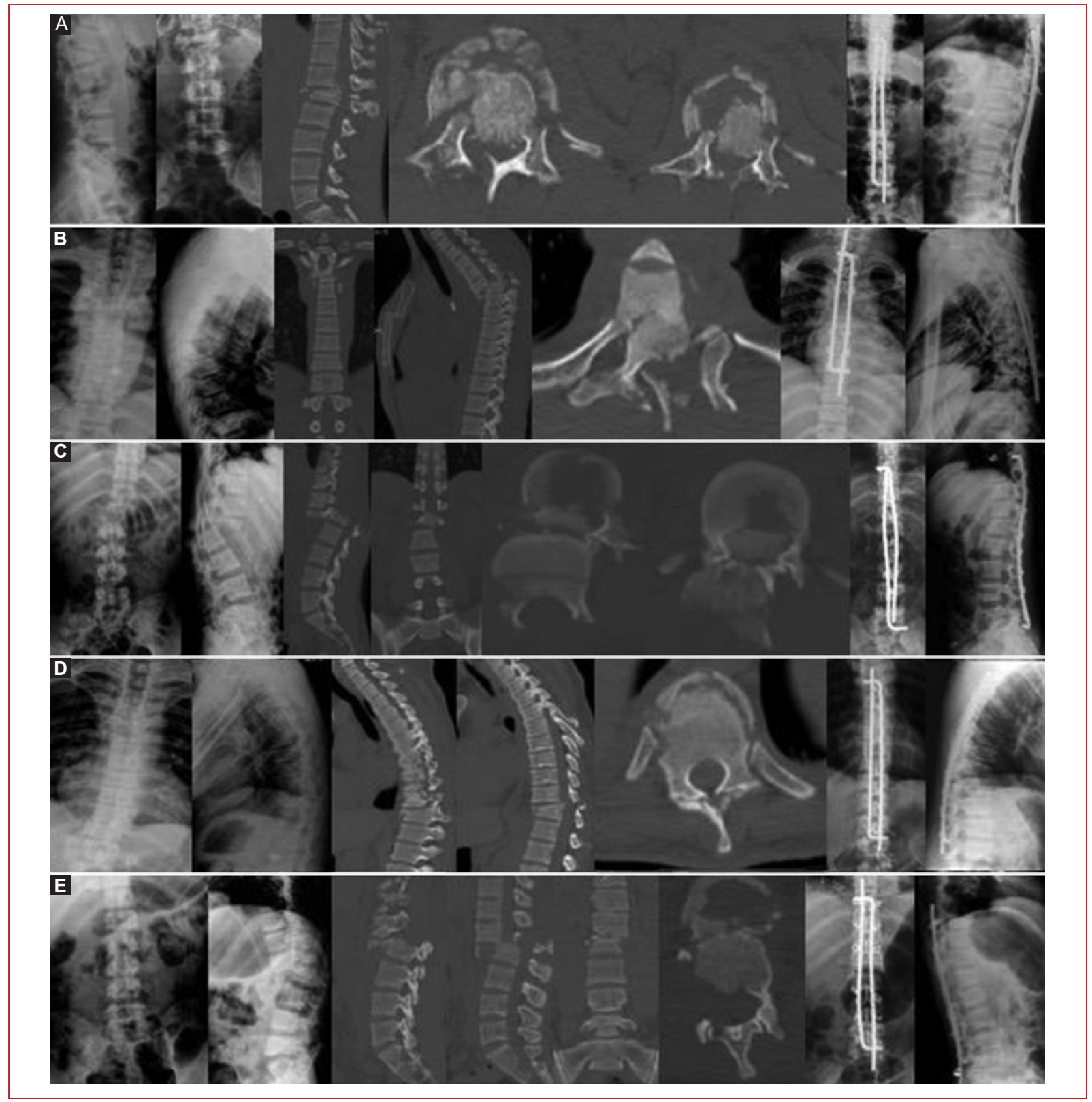

Figure 6. Cases of vertebral thoracolumbar fractures/dislocations treated in our center with the Luque instrumentation (A-E).

bracing is employed to help maintain fixation of the rods. Harrington rods are by design elongating the spine. Coronal plane correction is desirable, but in the sagittal plane, distraction forces could lead to a reduction of lumbar lordosis and cause flat-back syndrome ${ }^{17}$.

\section{Complications and limitations of the technique}

The major drawback to the Luque system is that the wires can impinge on the dura and spinal cord, causing neurological injury. There are reports of important neurological complications with the passing of the sublaminar wires and possible medullar damage up to $17 \%, 7,11$, and it is felt that there is a learning curve with the technique and decreased risk of complications over time for each surgeon ${ }^{18}$. However, this is not observed in the case of the authors' institution, where since 1976 we have used the system devised by Luque first for the treatment of paralytic scoliosis and Potts disease, and then for fractures and degenerative diseases, obtaining good results in selected cases.

Nevertheless, post-operative complications include infection, paresthesia, muscular weakness and pseudarthrosis, implant failure with the rupture of the wires or a 
fracture of the bars, leading to the loss of the correction, and immunological reactions to the implants ${ }^{18}$ (Fig. 5).

The previous studies have determined that Luque rod instrumentation with segmented sublaminar wiring is a more difficult and potentially dangerous procedure. However, with care and experience, good results can be achieved with few complications. The main advantages of Luque rods are that they maintain the normal sagittal contour, especially in the thoracolumbar and lumbar regions, and prevent loss of correction better than Harrington rods. The greater rigidity provided by segmental sublaminar wiring allows the patient to be mobilized within a few days of operation without external support. The main disadvantage of the method is that it has very little effect on derotating the spine, especially in the thoracic region ${ }^{19}$.

At present, transpedicular instrumentation techniques have become the gold standard for the treatment of deformities and fractures of the thoracic and lumbar spine. These provide biomechanical advantages superior to the Luque instrumentation, the need for less invasive approaches, a faster recovery, and a shorter hospital stay, mainly due to the more rigid internal fixation that they produce. However, they have the inconvenience of a high cost and are not accessible to the whole population. In the same way, the current transpedicular fixation systems have a lower rate of implant complications or failures, and a shorter learning curve compared to the Luque instrumentation ${ }^{20}$.

The most important current indication for the use of Luque's instrumentation is neuromuscular scoliosis, where it continues to have a relevant role in the management of this type of patient ${ }^{21}$.

\section{Conclusions}

At the present time, teaching the most current vertebral fixation and instrumentation systems to Mexican residents of orthopedic surgery is imperative. However, our actual health system, in which patients without medical coverage have high complexity traumatic or congenital spinal pathologies, has led to the teaching and implementation in some cases of the system developed by Dr. Luque almost five decades ago in Mexico City. Nowadays, between 5 and 10 procedures are performed using this technique in our hospital per year, reaching around 300 procedures performed between 1976 and 2018 (Fig. 6).

\section{Conflicts of interest}

The authors declare that they have no financial support and conflicts of interest.

\section{Funding}

No funding was received for the development of the study.

\section{Ethical disclosures}

Protection of human and animal subjects. The authors declare that no experiments were performed on humans or animals for this study.

Confidentiality of data. The authors declare that they have followed the protocols of their work center on the publication of patient data.

Right to privacy and informed consent. The authors have obtained the written informed consent of the patients or subjects mentioned in the article. The corresponding author is in possession of this document.

\section{References}

1. Luque ER. Segmental spinal instrumentation for correction of scoliosis. Clin Orthop Relat Res. 1982;163:192-8.

2. Rebollar EL. Profesores de la Ortopedia Mexicana. Profesor Eduardo Luque Rebollar. Orthotips. 2005;1:70-1. Available from: http://www.medigraphic.com/cgi-bin/new/resumenl.cgi?IDARTICULO=5197. [Last accessed on 2018 Sep 06].

3. Luque ER. Osteotomias de columna, nuevos conceptos. An Ortop Traumatol. 1976:12:105.

4. Luque ER. Estudio clínico patológico de la anatomía de la escoliosis. Acta Ortop. Latinoam. 1975;2:182.

5. Luque ER. Anatomy of scoliosis and its correction. Clin Orthop. 1974;105:298

6. Luque ER. The anatomic basis and development of segmental spinal instrumentation. Spine. 1982;7:256-9.

7. Luque ER Segmental Correction and Fixation of the Spine, Scientific Exhibit. Dallas, Texas: Fourty-Fifth Annual Meeting of the AAOS; 1978.

8. Gersoff WK, Renshaw TS. The treatment of scoliosis in cerebral palsy by posterior spinal fusion with luque-rod seg-mental instrumentation. J Bone Joint Surg Am. 1988;70:41-4.

9. Luque ER. Segmental Spinal Instrumentation. United States, Washington DC: Library of Congress Catalog Card; 1984. p. 86-9.

10. Cybulski GR, Von Roenn KA, D'Angelo CM, DeWald RL. Luque rod stabilization for metastatic disease of the spine. Surg Neurol. 1987;28:277-83.

11. Luque ER. Segmental correction of scoliosis with rigid internal fixation, preliminary report. Orthop Trans. 1977;1:136-7.

12. Luque ER. Treatment of scoliosis without external support. Orthop Trans. 1977;1:37.

13. Raney EM. Hooks and wires tried and true plus how to: POSNA1-DayCourse, April 29, 2009. J Pediatr Orthop. 2011;31:S81-7.

14. Hasler CC. A brief overview of 100 years of history of surgical treatment for adolescent idiopathic scoliosis. J Child Orthop. 2013;7:57-62.

15. Lonstein JE, Koop SE, Novachek TF, Perra JH. Results and complications after spinal fusion for neuromuscular scoliosis in cerebral palsy and static encephalopathy using luque galveston instrumentation: experience in 93 patients. Spine (Phila Pa 1976). 2012;37:583-91.

16. Harrington PR. Treatment of scoliosis: correction and internal fixation by spine instrumentation. J Bone Joint Surg Am. 1962;44:591-634.

17. Bridwell KH. Spinal instrumentation in the management of adolescent scoliosis. Clin Orthop Relat Res. 1997;335:64-72.

18. Wilber RG, Thompson GH, Shaffer JW, Brown RH, Nash CL Jr. Postoperative neurological deficits in segmental spinal instrumentation. A study using spinal cord monitoring. J Bone Joint Surg Am. 1984; 66:1178-87

19. Canavese F, Rousset M, Le Gledic B, Samba A, Dimeglio A. Surgical advances in the treatment of neuromuscular scoliosis. World $\mathrm{J}$ Orthop. 2014;5:124-33.

20. Rosner MK, Polly DW Jr., Kuklo TR, Ondra SL. Thoracic pedicle screw fixation for spinal deformity. Neurosurg Focus. 2003:14:e7.

21. McCarthy RE. Management of neuromuscular scoliosis. Orthop Clin North Am. 1999;30:435-49, 98 